\title{
Designing of Face Image Processing Technique for Sorting Out Japanese Raccoons Form Raccoons
}

\author{
Tadasuke Furuya $^{1}$, Yayoi Kaneko ${ }^{2}$, Hiroaki Ishii ${ }^{2}$, and Takafumi Saito ${ }^{2}$ \\ ${ }^{1}$ Tokyo University of Marine Science and Technology \\ tfuruya@kaiyodai.ac.jp \\ ${ }^{2}$ Tokyo University of Agriculture and Technology \\ txsaito@cc.tuat.ac.jp
}

\begin{abstract}
To observe mode of life of wild animals, it needs to research individual animal continually. We have to capture these animals temporarily. We need to capture them safely, of course, without hurting them. In general, we make use of traps and capture wild animals. Automation and remote-control operation which monitor traps are needed to reduce costs such as labor costs related to capture for long periods of time. For this reason we developed new type of traps that is possible of remote monitoring and remote operation over the network. Furthermore, we try to recognize face image toward automatic discrimination. In this study, we explain about preprocessing that face recognition is needed.
\end{abstract}

Keywords: animal trap, mobile telephone, non-photo realistic.

\section{Introduction}

To observe mode of life of wild animals, it needs to research individual animal continually. We observe mode of life of Japanese raccoons and raccoons at the present day. Therefore, we have to capture these animals temporarily. We need to capture them safely, of course, without hurting them. In general, we make use of traps and capture wild animals. However, there are roughly five problems about the capture by this trap.

These are 1)capture of error, 2)well-being of captive animals, 3)security of workers, 4)misclassification of captive animals, 5)high cost related to maintenance of traps. Recently, utilization of traps which have an effect of preventing capture of error, for example, egg traps and traps with a trigger of preventing by catch is carried forward. Automation and remote-control operation which monitor traps are needed to reduce costs such as labor costs related to capture for long periods of time.

\section{Trap}

In this study, we developed new type of traps (Fig.1) that is possible of remote monitoring and remote operation over the network. 
This trap carries communications device (Fig.2), mobile network, IP camera and remote door open system. We operate from browsing software like general pc and cellular phone over the network and take images in the trap. We can also monitor and remote-control the door of the trap open. After opening the door, it is possible to reset the trigger (Fig.3).

We indicate specifications of controlled box. We set cages at distant place and check connection of networks. When animals enter the cages, we receive notice by twitter. We check images of our cellular phone, then we open the cages if we capture animals excepting for raccoons. It is often difficult for distinguishing animals for the reason that captured images are small or are not bright etc. Also, we sometimes cannot send high-quality images in succession for the reason of communications line velocity or camera memory shortage. So, we generate images that humans can understand shot animals in a short time on condition that there are a small number of images, contents are not recognized and brightness is not controlled. Furthermore, a system that automatically recognize what kind of animal it is among pictures of animal's faces and automatically open cages is expected. It is difficult for us to recognize faces of animals shot outside. Then, in this study, we consider preprocessing toward recognition.

The trap makes us possible to detect captured animals at an early date, deal with the matter appropriately and prevent them from injuries and weakness. We can also simultaneously monitor several traps in a remote location and decrease frequency of going in for a look-around. Therefore, it leads to cost reduction of labor costs. In addition to this, it is possible for a few specialists to monitor several regions and several traps at one time. Then, they can discriminate among captured animals. In the end, the trap leads to the prevention of killing captured individuals by mistake.

Moreover, if the starting time we operate the traps can be shorten, wild animals are less affected by the stress.

To realize this, it is needed to the equipment which is classified faces of animals and automatically let go of animals that we don't want to observe. In this study, we consider the method of image processing related to distinguish Japanese raccoons from raccoons.

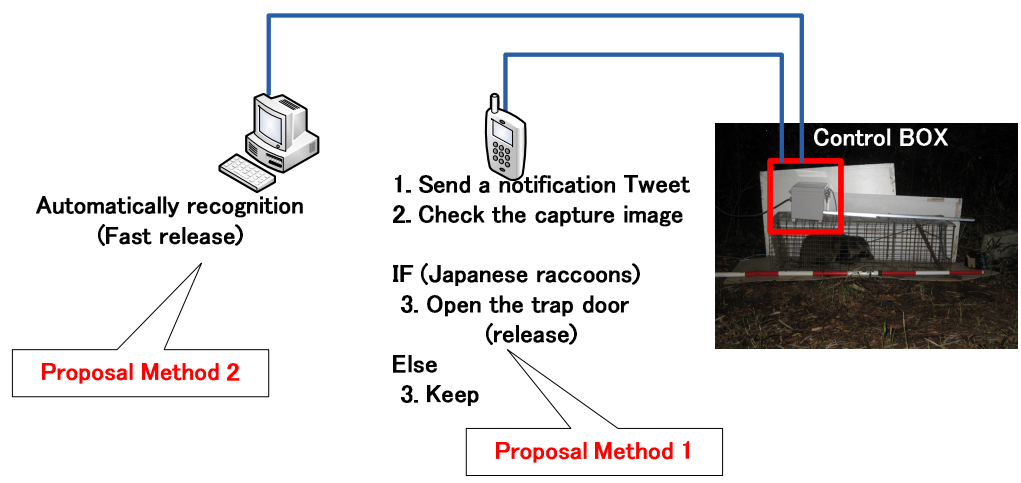

Fig. 1. Trap system 


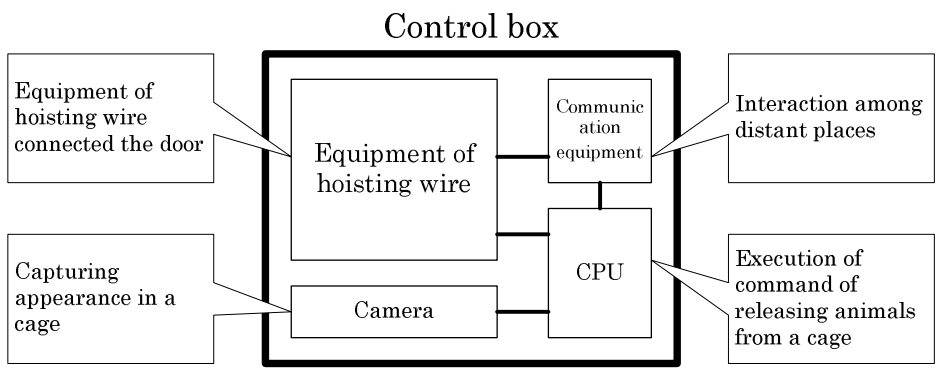

Fig. 2. Control Box

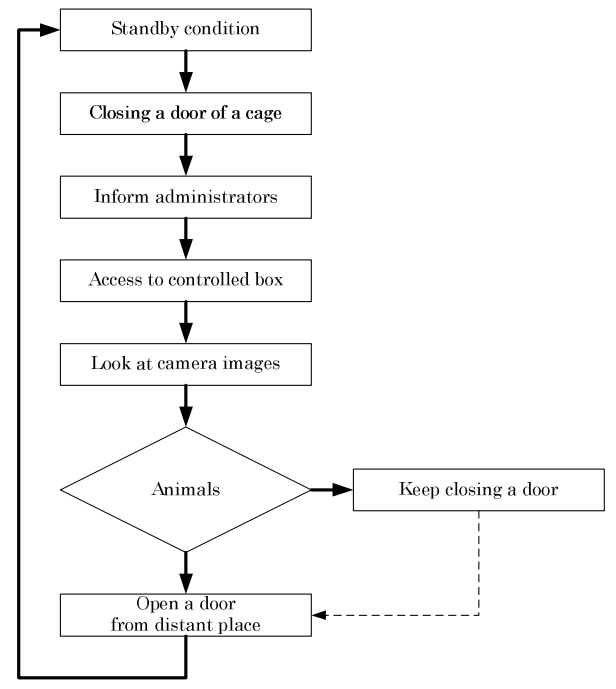

Fig. 3. System Flow

\section{Proposed Method}

It is difficult to extract a part of feature from faces of animals covered with fur. The images pictured outside aren't also available images to judge animals sufficiently. Under the conditions, it is needed to emphatically display the figure of faces. Generally, space filter and concentration conversion are often utilized. In this method, though processing speed to combine with easy image processing is fast, it is difficult to express the images sophisticatedly due to manipulate all of the images uniformly. So, we consider examine the environment for the usage of the trap, and examine the method independent of lighting condition without interactive operation.

It is hard to use color information of shooting images because the animals made the color of their fur change with the seasons. In addition it is difficult to judge whether a part of shooting images is pattern or shadow. Then, we make rough texture information and images we can recognize the direction of their fur. 
In this study, we attempt to emphasize distinctive line of the animals through the use of multi scale analysis. By combining with several variously-sized Gaussian filters, we do frequency decomposition and recombine weight for each band-pass images, then we control systematic level of detail at each band.

1. Generation of gray-scale images from captured images(Fig.2,5) and normalization brightness value

2. Generation of different scale of smoothing images

3. Acquisition of band images from difference of neighboring scale of smoothing images

4. Addition of adequate bias value

5. Mapping on adequate brightness coverage from obtained brightness value

In this method, we generate the composite images that leave quite characteristic of the animals clearly and detail characteristic of them. At this time, we make out two types of images that change the rule of weight. We read the features of Japanese raccoons and raccoons through these two types of images. Generally speaking, raccoons contain a black line between their eyebrows, on the other hand, Japanese raccoons don't contain that. The verge of raccoon's ears also is white, but that of Japanese raccoon's ears is black. From an examination of image processing, we can understand their characteristics which are displayed from the composite images. We can also obtain the stable consequence without depending on lighting conditions.

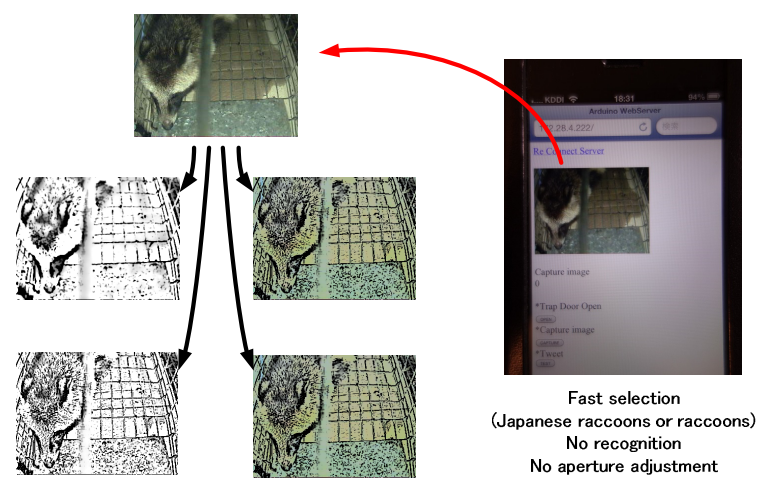

Fig. 4. Cellular phone screen

\section{Conclusion}

For the future, we try to automatically select face of raccoons and that of Japanese raccoons by extracting the features of their eyes, nose and mouth from images based on this method or by mapping on the characteristic space. 